\title{
The luminosity distribution of RSGs to test their mass-loss rate
}

\author{
Cyril Georgy $^{1,2}$ and Sylvia Ekström ${ }^{1}$ \\ ${ }^{1}$ Geneva Observatory, Geneva University \\ Chemin des Maillettes 51, 1290 Versoix, Switzerland \\ email: cyril.georgy@unige.ch \\ email: sylvia.ekstrom@unige.ch \\ ${ }^{2}$ Astrophysics Group, Keele University \\ Keele, ST5 5BG, United Kingdom
}

\begin{abstract}
The red supergiant phase is an important phase of the evolution of massive star, as it mostly determines its final stages. One of the most important driver of the evolution during this phase is mass loss. However, the mass-loss rates prescription used for red supergiants in current stellar evolution models are still very inaccurate.

Varying the mass-loss rate makes the star evolve for some time in yellow/blue regions of the HRD, modifying the number of RSGs in some luminosity ranges. Figure 1 shows how the luminosity distribution of RSGs is modified for various mass-loss prescriptions. This illustrates that it is theoretically possible to determine at least roughly what is the typical mass loss regime of RSGs in a stellar evolution perspective.
\end{abstract}

Keywords. Stars: mass-loss, Stars: supergiants, Stars: luminosity function

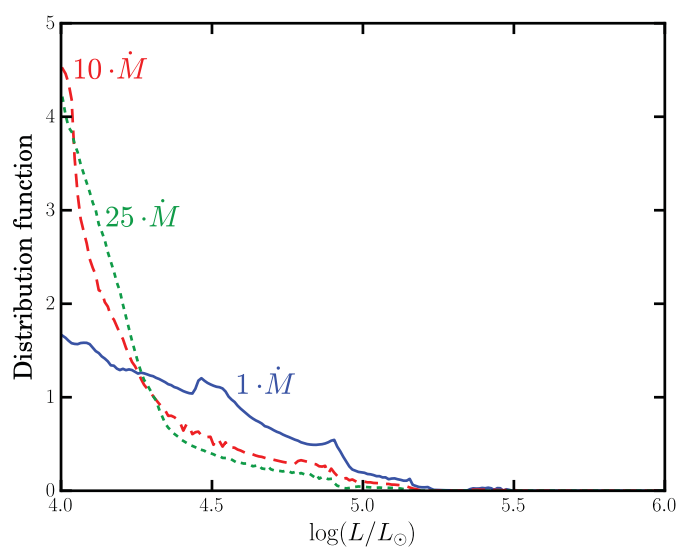

Figure 1. Luminosity distribution of solar metallicity RSGs assuming various mass-loss rates: standard one (solid blue line), 10 times standard (dashed red line) and 25 times standard (dotted green line).

\section{Acknowledgements}

CG acknowledges support from the European Research Council under the European Union's Seventh Framework Programme (FP/2007-2013) / ERC Grant Agreement n. 306901. 